\title{
Downregulation of Bmi-1 suppresses epithelial-mesenchymal transition in melanoma
}

\author{
YANTING LIU ${ }^{1}$, ZHAOWEI CHU ${ }^{1}$, QINGYAN LI ${ }^{1}$, BIN PENG $^{1}$, SUYUN XU ${ }^{2}$, \\ CHRISTINE G. LIAN ${ }^{2}$ and SONGMEI GENG ${ }^{1}$ \\ ${ }^{1}$ Department of Dermatology, Northwest Hospital, Xi'an Jiaotong University, Xi'an, Shaanxi 710004, P.R. China; \\ ${ }^{2}$ Department of Pathology, Brigham and Women's Hospital, Harvard Medical School, Boston, MA 02115, USA
}

Received May 30, 2016; Accepted July 8, 2016

DOI: $10.3892 /$ or.2016.5244

\begin{abstract}
Epithelial-mesenchymal transition (EMT) contributes to the invasion and metastasis of numerous malignant cancers, including melanoma. A significant higher expression of B-lymphoma Moloney murine leukemia virus insertion region-1 (Bmi-1) has been reported in cell lines from metastatic melanoma compared to cell lines from primary melanoma. There are studies that show that knockdown of Bmi-1 could induce E-cadherin expression in melanoma cells. However, the role of Bmi-1 in mediating EMT-like changes in melanoma has not yet been fully studied. In the present study, knockdown of Bmi-1 by shRNA transduction decreased the invasion properties of the cultured human melanoma cells A375 by a Matrigel invasion assay, along with alterations in EMT-related markers E-cadherin, $\alpha$-catenin, vimentin and $\mathrm{N}$-cadherin. The aforementioned altered expression of EMT markers was verified in BALB/c-nude mouse xenografts. Furthermore, to explore the underlying regulatory mechanism of EMT, we detected the significant downregulation of $\mathrm{p}-\mathrm{Akt} / \mathrm{p}-\mathrm{NF}-\kappa \mathrm{B} / \mathrm{MMP}-2$ and the upregulation of PTEN in Bmi-1-silenced A375 cells. The present study demonstrated that knockdown of Bmi-1 significantly inhibited the aggressive behavior of melanoma by reversing EMT-like changes via the PTEN/p-Akt/p-NF-кB/MMP-2 pathway.
\end{abstract}

\section{Introduction}

Despite significant scientific innovations in cancer treatments over the last few decades, melanoma remains one of the most

Correspondence to: Professor Christine G. Lian, Department of Pathology, Brigham and Women's Hospital, Harvard Medical School, 221 Longwood Ave., Boston, MA 02115, USA

E-mail: cglian@bwh.harvard.edu

Professor Songmei Geng, Department of Dermatology, Northwest Hospital, Xi'an Jiaotong University, $157 \mathrm{Xi}$ Wu Road, Xi'an, Shaanxi 710004, P.R. China

E-mail:gsm312@yahoo.com

Key words: Bmi-1, epithelial-mesenchymal transition, melanoma, PTEN, NF- $\mathrm{KB}$ aggressive malignant tumors, with increasing incidence, poor prognosis and insensitivity to chemotherapy (1-3). Melanoma is extremely difficult to treat once it has metastasized with a short 5-year survival rate (4). The phenomenon of epithelial-mesenchymal transition (EMT) with characteristic changes in the loss of epithelial markers (E-cadherin and $\alpha$-catenin) and the gain of mesenchymal markers (vimentin and $\mathrm{N}$-cadherin) have been well documented in cancer invasion and metastasis (5-9).

B-lymphoma Moloney murine leukemia virus insertion region-1 (Bmi-1), the first member of the polycomb group (PcG) gene family identified in mammals, plays an essential role in the self-renewal of stem cells and functions as an oncogene in human malignancies as well (5,6,8,10-12). Bmi-1 was found to be highly expressed in cell lines from metastatic melanoma compared to cell lines from primary melanoma, and may be relevant for the metastatic behavior of melanoma cells (13). The rapidly growing information shows that Bmi-1 overexpression can induce EMT properties and promote the metastasis of cancers, such as nasopharyngeal carcinoma, endometrial and breast cancer $(6,8,10)$. In cultured human melanoma WM115A cells, it was reported that silencing of Bmi-1 induced upregulation of E-cadherin, one of the key epithelial markers of EMT (14). This evidence suggests that overexpression of Bmi-1 induces EMT-like changes and promotes metastasis in melanoma. However, the effects of Bmi-1 on EMT-like changes, invasion and proliferation of melanoma have not been fully addressed and the underlying mechanism of Bmi-1 mediating EMT-like changes in melanoma remains currently unclear.

Nuclear factor- $\kappa \mathrm{B}(\mathrm{NF}-\kappa \mathrm{B})$, as a transcriptional factor, regulates the transcription of genes in several critical pathways in cancer biology, such as apoptosis, angiogenesis, proliferation and tumor cell invasion and metastasis $(4,15)$. Several studies show that activation of the NF- $\kappa \mathrm{B}$ pathway directly regulates EMT in melanoma (4). Moreover, the activation of $\mathrm{NF}-\kappa \mathrm{B}$ could lead to the inhibition of phosphatase and tensin homolog (PTEN) transcription in non-small cell lung cancer cells (16). The tumor-suppressor PTEN plays a significant role in cell cycle arrest, regulation of cell adhesion, migration and proliferation (4). It has been reported that PTEN participates in Bmi-1-induced EMT in human nasopharyngeal epithelial cells (8). Hereby, whether NF- $\kappa \mathrm{B}$ and PTEN participate in 
Bmi-1-induced EMT-like changes in melanoma cells have not been fully addressed.

The present study focused on the effects of the silencing of Bmi-1 on EMT-like changes in cultured melanoma cells in vitro and on a human melanoma mouse xenograft model in vivo. We demonstrated that Bmi-1 knockdown reduced melanoma cell invasion detected by Matrigel invasion assay and tumor growth evaluation in xenografts, respectively. Increased expression of epithelial marker genes and decreased expression of mesenchymal marker genes indicate the reversal of EMT-like changes induced by silencing of Bmi-1 in melanoma. In addition, knockdown of Bmi-1 led to the suppression of NF- $\mathrm{B}$ and MMP-2 while significantly upregulating PTEN. Collectively, these results provide evidence that knockdown of Bmi-1 significantly inhibited the aggressive behavior of melanoma cells through the reversal of EMT-like changes via the regulation of related genes of the $\mathrm{NF}-\kappa \mathrm{B}$ and PTEN pathways.

\section{Materials and methods}

Cell culture. The melanoma cell lines A375, C8161, SK-Mel-5 and Mel-Juso were cultured in Dulbecco's modified Eagle's medium (DMEM) supplemented with $10 \%$ fetal bovine serum (Gibco, Grand Island, NY, USA). All cultures were maintained in a $5 \% \mathrm{CO}_{2}$ incubator at $37^{\circ} \mathrm{C}$. After detecting the Bmi-1 levels in the aforementioned cell lines, the optimal cells were selected for further study.

Bmi-1 silencing by shRNA. The siRNA target sequence for the Bmi-1 gene (NM_005180) was designed and the si-Bmi-1 constructs were inserted into the GV248 green fluorescent protein-expressing lentiviral vector (GeneChem, Shanghai, China). After a series of programmes to transfect, concentrate and purify, Bmi-1 shRNA lentivirus and empty lentivirus were obtained with a range of titer yields: $1-1.5 \times 10^{9} \mathrm{TU} / \mathrm{ml}$ and transfected into A375 cells (MOI=20). The expression of Bmi-1 in A375 cells after transfection was confirmed by real-time PCR and western blot analysis.

Real-time quantitative RT-PCR. Total RNA from A375, Bmi-1-knockdown (A375-Bmi-1-shRNA) and pMSCV vector control cells (A375-vector) were extracted using the RNeasy Midi kit (Qiagen, Inc., Valencia, CA, USA) and reverse transcribed to cDNA with a PrimeScript RT reagent kit (Takara, Tokyo, Japan). Real-time PCR was performed using SYBR Premix Ex Taq (Takara) on a StepOnePlus ${ }^{\mathrm{TM}}$ Real-Time PCR System (Applied Biosystems, Invitrogen). The cycling conditions were as follows: $95^{\circ} \mathrm{C}$ for $30 \mathrm{sec}, 95^{\circ} \mathrm{C}$ for $10 \mathrm{sec}$ and $60^{\circ} \mathrm{C}$ for $30 \mathrm{sec}$. The following are the gene-specific primer pairs: Bmi-1, 5'-CTGGTTGCCCATTGACAGCG-3' and 5'-AAATCCCGGAAAGAGCAGCC-3'; E-cadherin, 5'-CGG TGGTCAAAGAGCCCTTA-3' and 5'-TGAGGGTTGGTGC AACGTCGTTA-3'; $\alpha$-catenin, 5'-GGCAGCCAAAAGACAA CAGG-3' and 5'-GGCCTTATAGGCTGCGACAT-3'; vimentin, 5'-CAGGCAAAGCAGGAGTCCAC-3' and 5'-GCA GCTTCAACGGCAAAGTTC-3'; N-cadherin, 5'-CGA ATGGATGAAAGACCCATCC-3' and 5'-GCCACTGCCTT CATAGTCAAACACT-3'; and $\beta$-actin, 5'-TGGCACCCAGC ACAATGAA-3' and 5'-CTAAGTCATAGTCCGCCTAGA
AGCA-3'. The gene expression levels were calculated using the $2^{-\Delta \Delta \mathrm{Ct}}$ method.

Western blot analysis. Total protein was extracted from the aforementioned cultured cells and quantified. Equal protein amounts were analyzed using 10\% SDS-PAGE and transferred to a polyvinylidene difluoride membrane. After blocking with a non-specific antibody binding with non-fat dried milk for $2 \mathrm{~h}$ at room temperature, the membranes were incubated overnight with primary antibodies at $4{ }^{\circ} \mathrm{C}$ and then incubated with a horseradish peroxidase-conjugated secondary antibody for $2 \mathrm{~h}$ at room temperature. The primary antibodies used were: anti-Bmi-1 (Abcam, Cambridge, UK), anti-E-cadherin, anti- $\alpha$-catenin (both from ProteinTech, Chicago, IL, USA), anti-vimentin, anti-N-cadherin (both from Zhongshan Co., Beijing, China), anti-PTEN (Santa Cruz Biotechnology, Inc., Santa Cruz, CA, USA), anti-Akt, anti-phospho-Akt (both from Cell Signaling Technology, Inc., Beverly, MA,

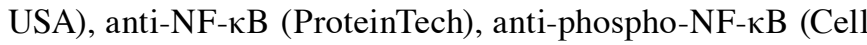
Signaling Technology, Inc.), anti-MMP-2 (ProteinTech) and anti- $\beta$-actin (Santa Cruz Biotechnology, Inc.).

Immunofluorescent staining. The cells were fixed in $4 \%$ paraformaldehyde, permeabilized in a solution of $0.3 \%$ Triton X-100 and 5\% bovine serum albumin (BSA) for $30 \mathrm{~min}$ at room temperature and incubated with antibodies against E-cadherin, $\alpha$-catenin, vimentin or $\mathrm{N}$-cadherin overnight at $4^{\circ} \mathrm{C}$. Subsequently the cells were incubated with the appropriate $\mathrm{Cy} 3$-conjugated secondary antibody (Jackson ImmunoResearch Laboratories, West Grove, PA, USA) at $37^{\circ} \mathrm{C}$ for $1 \mathrm{~h}$. The nuclei were counterstained with DAPI and imaged with a confocal laser-scanning microscope (Nikon, Tokyo, Japan).

Immunohistochemistry. After dewaxing and rehydration, the sections were subjected to antigen retrieval by boiling in $10 \mathrm{mM}$ citrate buffer. After incubation with $3 \%$ hydrogen peroxide for 20 min to block the endogenous peroxidase activity, the sections were immersed in $5 \%$ bovine serum for $30 \mathrm{~min}$ to minimize antibody non-specificity. Next, the sections were incubated with antibodies (anti-E-cadherin, anti- $\alpha$-catenin, anti-vimentin and anti-N-cadherin) overnight at $4^{\circ} \mathrm{C}$ and then washed. The sections were then treated with secondary antibodies (horseradish peroxidase-labeled goat anti-mouse/rabbit; Zhongshan Co.) for $30 \mathrm{~min}$ at $37^{\circ} \mathrm{C}$. Following development by diaminobenzidine (Zhongshan Co.), the sections were counterstained with hematoxylin and evaluated using microscopy.

Matrigel invasion assays. The Transwell polycarbonate membranes containing $8-\mu \mathrm{m}$ pores were coated with Matrigel (BD Biosciences, San Jose, CA, USA). Cells were resuspended in serum-free DMEM and seeded into the upper wells, and DMEM supplemented with $15 \%$ bovine serum was placed into the lower chamber (6). The cells that migrated through the membranes were observed with crystal violet staining after incubation for $24 \mathrm{~h}$ at $37^{\circ} \mathrm{C}$.

Xenograft tumors. Six-week-old female BALB/c-nude mice were obtained. Animals were housed in pathogen-free 
A

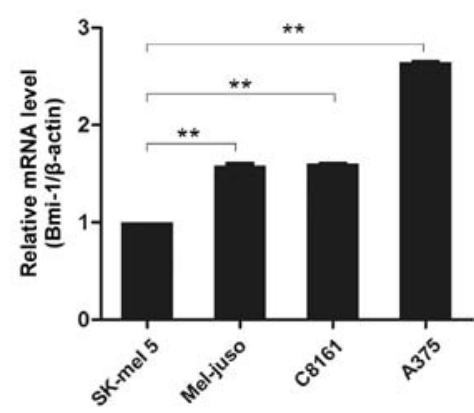

C

$\mathbf{E}$
B

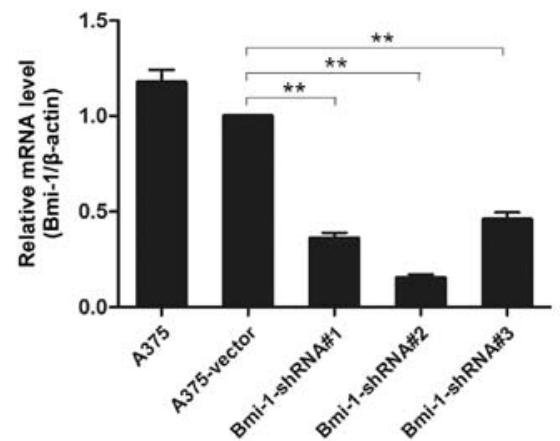

D

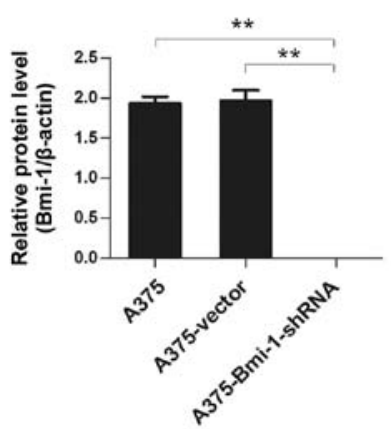

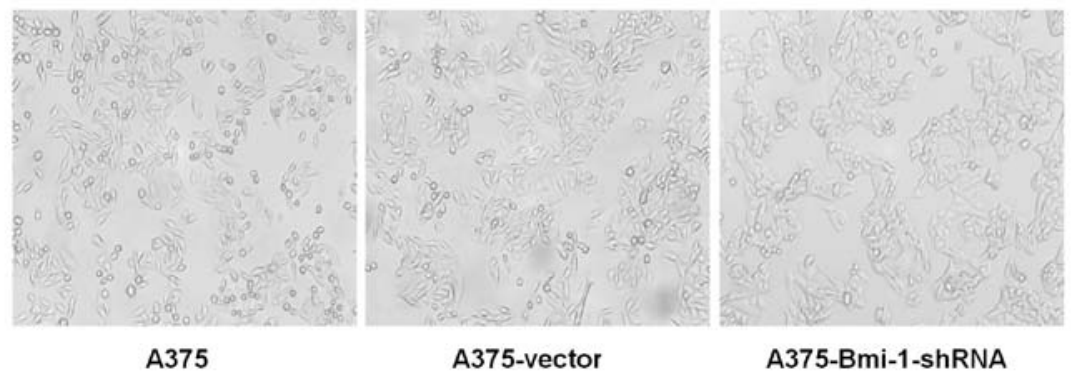

Figure 1. Silencing of Bmi-1 reduces A375 cell invasion. (A) The expression level of Bmi-1 in several melanoma cells. A higher Bmi-1 level was detected in the A375 cell line, which was selected for further study. (B) Quantitative RT-PCR analysis of Bmi-1 expression in the A375 cell lines. All three shRNAs specifically knocked down endogenous Bmi-1 mRNA. shRNA \#2 was chosen for subsequent studies. (C) Western blot analysis of Bmi-1 protein in the A375 cell lines. (D) Quantitation of the data in C. The Bmi-1 protein of A375-Bmi-1-shRNA cell line was significantly silenced compared with the A375 cell line and the A375-vector cell line. Data are expressed as the means \pm SD (n=3). (E) Morphological observation showed that A375-Bmi-1-shRNA cells gathered more closely to each other, while normal A375 cells appeared spindle-like and had a fibroblastic morphology. ${ }^{* *} \mathrm{P}<0.001$.

conditions with filtered air, autoclaved food and water was available. A375-vector, A375-Bmi-1-shRNA and 4x10 cells in serum-free DMEM were injected subcutaneously into mice on their right flanks (six mice/group). Tumor xenografts were measured once/week with calipers. Mice were observed with live small-animal imaging technology at the end of the fifth week. Tumor xenografts were harvested for histological analysis. The volume was calculated as: $a b^{2} / 2$ (a and b represent the length and width of a tumor, respectively) and the tumor weights were measured. These data were statistically compared by a two-sample t-test, with a significance level of $\mathrm{P}<0.05$. The histology and immunohistochemistry of the tumors were observed under a microscope.

\section{Results}

Silencing of Bmi-1 in cultured human A375 cell line (A375-Bmi-1-shRNA). To investigate the impact of Bmi-1 on EMT, we compared the expression level of Bmi-1 in several melanoma cell lines, and the A375 cell line was selected for further study (Fig. 1A). We silenced endogenous Bmi-1 in the A375 cells using specific shRNAs and knockdown effects were detected by real-time PCR and western blotting. All three shRNAs specifically knocked down the mRNA levels of Bmi-1. Due to the best efficacy of shRNA \#2, it was chosen for subsequent studies (Fig. 1B). As shown in Fig. 1, Bmi-1 was significantly silenced both at the mRNA and protein levels (Fig. 1B-D).

Silencing of Bmi-1 reduces A375 cell invasion and reverses the EMT-like phenotype in vitro. Significant morphological differences were observed in the A375-Bmi-1-shRNA cells in that there were more cohesive cells with epithelioid changes compared to the spindle-shaped normal A375 cells (Fig. 1E). The A375-Bmi-1-shRNA cells gathered more closely to each other, while the normal A375 cells appeared as spindle-like with a fibroblastic morphology (Fig. 1E). Meanwhile, Matrigel invasion chamber assays revealed significantly reduced invasion of the A375-Bmi-1-shRNA cells when compared to the control cells (Fig. 2D and E). 
A

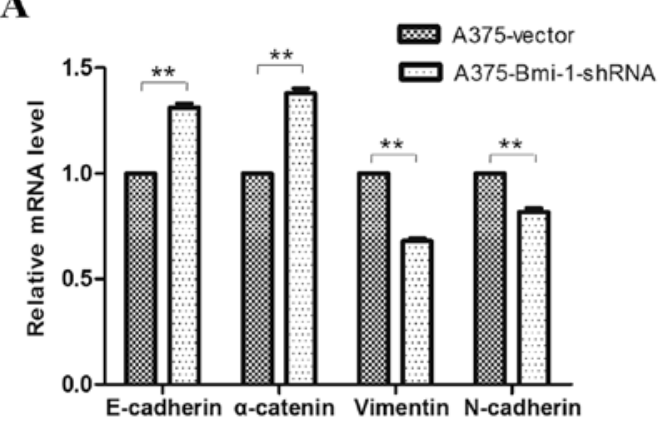

B

D

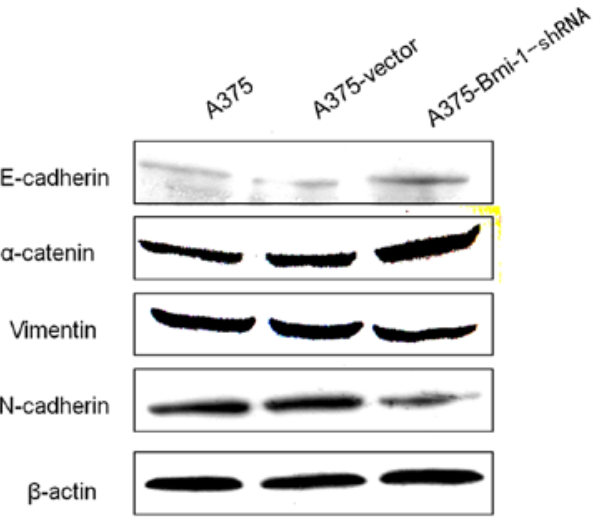

$\mathbf{E}$

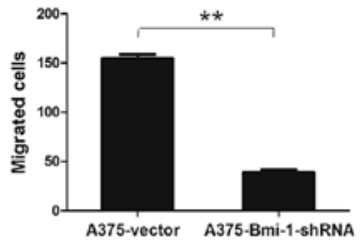

$\mathrm{N}$-cadherin

a-catenin

Vimentin

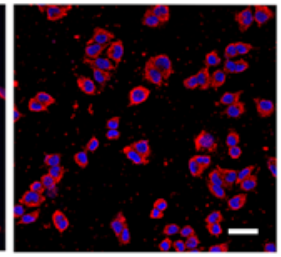

-
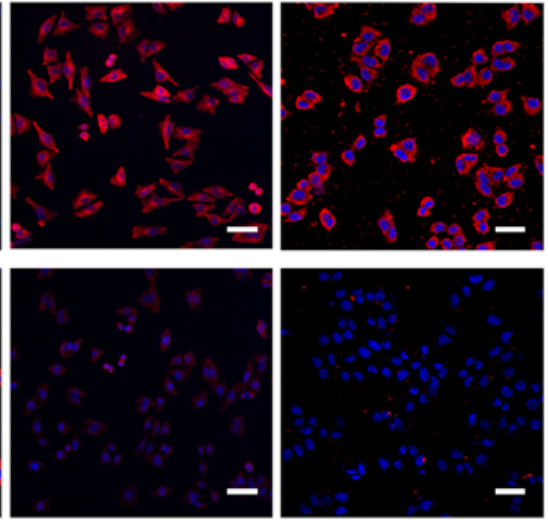

Figure 2. Silencing of Bmi-1 reverses the EMT phenotype. (A) Quantitative RT-PCR analysis of the expression of epithelial and mesenchymal markers in the A375 cell lines. Silencing of Bmi-1 in the A375 cells led to significantly enhanced expression of epithelial markers (E-cadherin and $\alpha$-catenin) and concomitant significant decreases in the expression of mesenchymal markers (vimentin and N-cadherin). (B) Western blot analysis of EMT marker proteins in the A375 cell lines. (C) Quantitation of the data in B. In accordance with the aforementioned results, the expression of epithelial markers (E-cadherin and $\alpha$-catenin) increased and mesenchymal markers (vimentin and N-cadherin) decreased in the A375-Bmi-1-shRNA cell line compared to the A375-vector cell line. (D and E) Matrigel invasion chamber assays revealed that Bmi-1 silencing significantly reduced the invasiveness of the A375 cell line. (F) Immunofluorescent staining showed that the expression of epithelial markers (E-cadherin and $\alpha$-catenin) increased and that of mesenchymal markers (vimentin and $\mathrm{N}$-cadherin) decreased in the A375-Bmi-1-shRNA cell line compared to the A375-vector cell line. ${ }^{* *} \mathrm{P}<0.001$; bar, $50 \mu \mathrm{m}$.

EMT marker expression following the silencing of Bmi-1 in A375 cells was detected and showed increased expression of epithelial markers (E-cadherin and $\alpha$-catenin) with concomitantly decreased expression of mesenchymal markers (vimentin and $\mathrm{N}$-cadherin) compared to the A375 control cells (Fig. 2A-C and F).Thus, our results suggested that Bmi-1 knockdown was crucial to reverse EMT-like changes and suppress invasiveness of cultured A375 cells in vitro.

Silencing Bmi-1 supresses melanoma growth and reverses EMT-like changes in a human melanoma mouse xenograft model in vivo. To further evaluate the effect of Bmi-1 knockdown on tumor growth and EMT properties in vivo, six-week-old female BALB/c-nude mice were injected with the A375-vector and the A375-Bmi-1-shRNA cells in their flanks (six mice for each group). Both the A375-vector cells and the A375-Bmi-1-shRNA cells formed palpable tumors after 10 days, however, there was no marked difference between the two groups until the third week (Fig. 3B). Tumors in the control group grew quickly in the fourth and fifth week, while tumors in the A375-Bmi-1-shRNA group grew significantly slower with no visible substantial changes (Fig. 3B). The mice were observed for five weeks and the xenograft tumors were harvested. The tumor nodules formed by the A375-Bmi-1shRNA cells were significantly smaller than those formed by the A375 control cells at the end of the experiment 
A

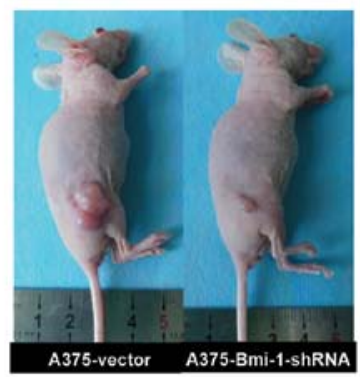

B

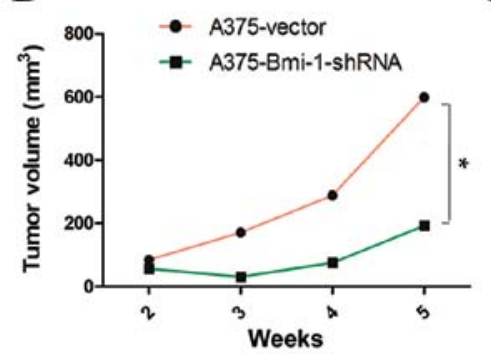

C

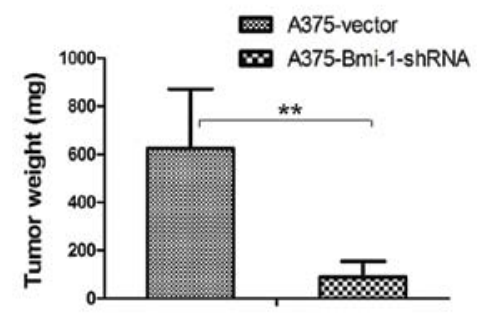

D

E-cadherin

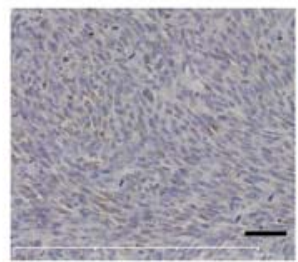

A375-vecto

A375-Bmi-1-shRNA

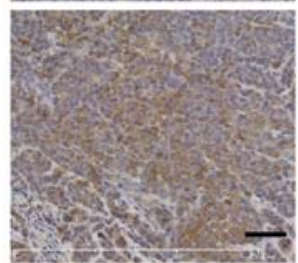

a-catenin

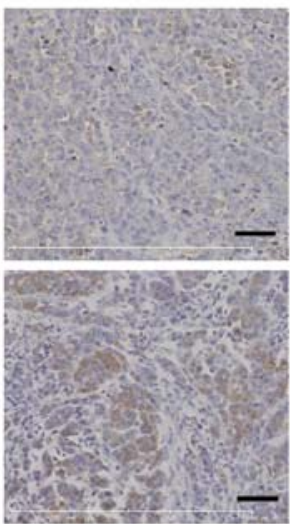

Vimentin

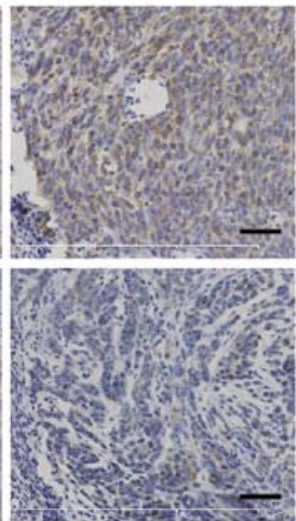

N-cadherin

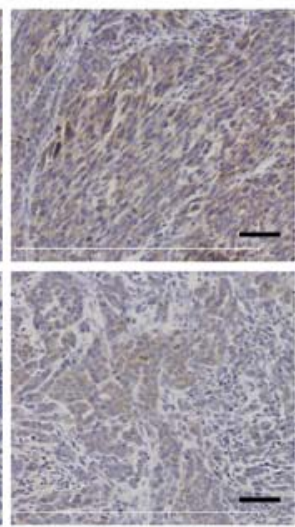

Figure 3. Silencing of Bmi-1 suppresses the proliferating abilities and EMT properties in vivo of melanoma. A375 control cells and A375-Bmi-1-shRNA cells were subcutaneously injected into the right flanks of mice. The mice were regularly observed for tumor growth. (A) The tumors formed by the A375-Bmi-1shRNA cells were significantly smaller than those formed by the A375 control cells at the end of the experiment (day 35 ). (B) There was no marked difference between the two groups of mice until the third week. Tumors in the control group grew quickly in the fourth and fifth week, while tumors in the Bmi-1-silenced group grew slowly with no visible changes. The average volume $\left(\mathrm{A} 375\right.$ control $=598.95 \pm 361.11 \mathrm{~mm}^{3}, \mathrm{~A} 375-\mathrm{Bmi}-1-\mathrm{shRNA}=192.17 \pm 164.26 \mathrm{~mm}^{3}, \mathrm{n}=6$, $\mathrm{P}=0.031)$ significantly differed between the two groups. (C) The average weight (A375 control $=624.2 \pm 246.8 \mathrm{mg}, \mathrm{A} 375-\mathrm{Bmi}-1-\mathrm{shRNA}=89.67 \pm 65.28 \mathrm{mg}$ $\mathrm{n}=6, \mathrm{P}=0.000$ ) significantly differed between the two groups. (D) Expression of EMT markers of tumorigenicity in mice. Compared with the tumors formed by the A375 control cells, the expression levels of E-cadherin and $\alpha$-catenin were upregulated while that of vimentin and $\mathrm{N}$-cadherin were downregulated in the tumors formed by the A375-Bmi-1-shRNA cells. ${ }^{*} \mathrm{P}<0.05$ and ${ }^{* *} \mathrm{P}<0.001$; bar, $50 \mu \mathrm{m}$.

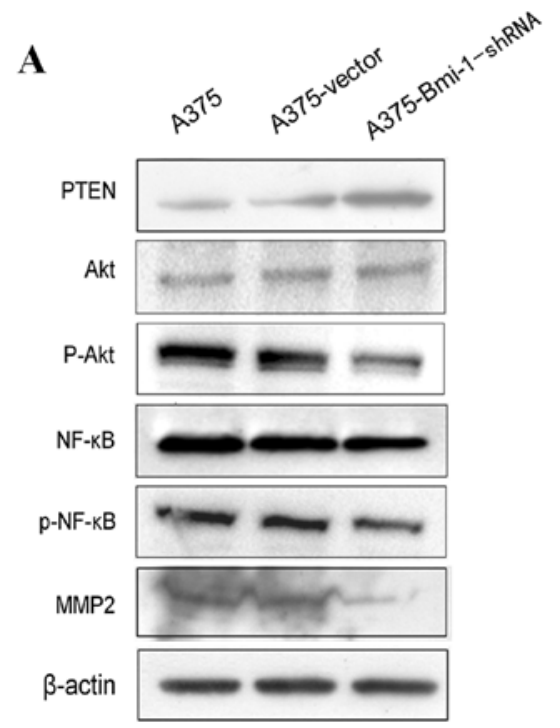

B

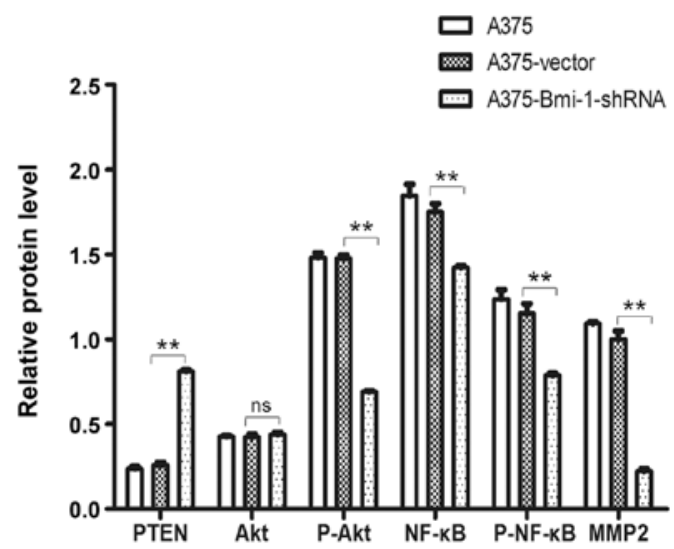

Figure 4. Knockdown of Bmi-1 suppresses melanoma invasion by regulating PTEN/Akt/NF- $\kappa$ B in the A375 cells. (A) Silencing of Bmi-1 upregulated its downstream target PTEN and then downregulated the protein expression of p-Akt, p-NF- $\mathrm{B}$ and MMP-2 in the A375 cells. (B) Quantitation of data in A. ${ }^{\mathrm{n} s} \mathrm{P}>0.05$ and ${ }^{* *} \mathrm{P}<0.001$.

(day 35) (Fig. 3A and B). As the data showed (Fig. 3B and C), the average volume $\left(\mathrm{A} 375-\right.$ vector $=598.95 \pm 361.11 \mathrm{~mm}^{3}$, A375-Bmi-1-shRNA $=192.17 \pm 164.26 \mathrm{~mm}^{3}, \mathrm{n}=6, \mathrm{P}=0.031$ ) and the average of the weight (A375-vector $=624.2 \pm 246.8 \mathrm{mg}$, A375-Bmi-1-shRNA $=89.67 \pm 65.28 \mathrm{mg}, \mathrm{n}=6, \mathrm{P}=0.000)$ had significant differences between the two groups. 


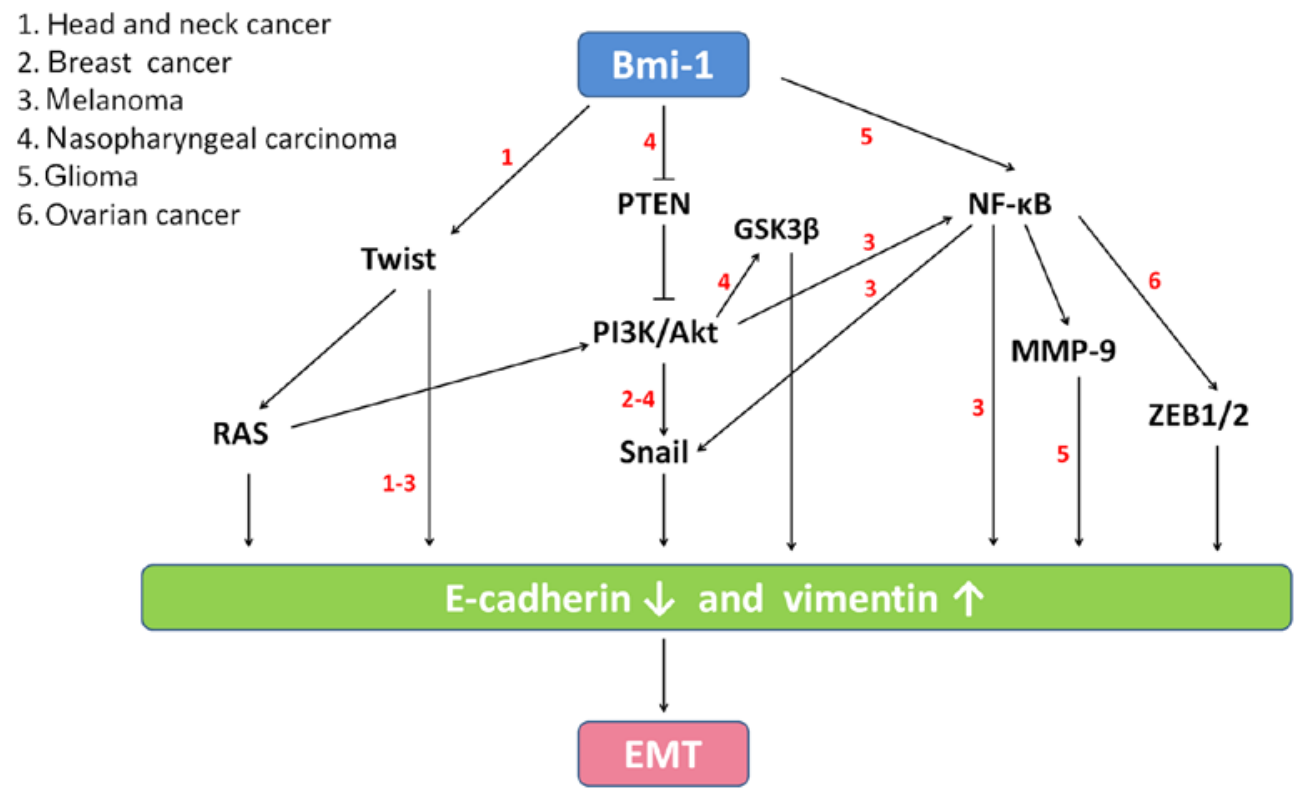

Figure 5. Pathways involved in the Bmi-1 regulation of EMT in various types of cancers.

To confirm that the silencing of Bmi-1 suppresses melanoma proliferative ability by reversing EMT tumorigenicity in mice, we evaluated the expression of EMT markers in tumor xenograft tissues. Compared with tumors formed by the A375vector cells, the expression levels of E-cadherin and $\alpha$-catenin were upregulated, while vimentin and $\mathrm{N}$-cadherin were downregulated in the tumors formed by the A375-Bmi-1-shRNA cells (Fig. 3D). The findings were consistent with the gene expression of EMT markers in the Bmi-1-silenced A375 cells.

Silencing of Bmi-1 reverses EMT-like changes via the upregulation of PTEN and downregulation of $p-A k t / p$ $N F-\kappa B / M M P-2$ in A375 cells. The tumor-suppressor PTEN, a downstream target of Bmi-1, was increased in the A375Bmi-1-shRNA cells, while its targeted downstream molecules p-Akt, p-NF- $\kappa \mathrm{B}$ and matrix metalloproteases-2 (MMP-2) were reduced when Bmi-1 was knocked down (Fig. 4). On the one hand, activation of Akt activated the NF- $\kappa \mathrm{B}$ pathway, which promoted EMT in melanoma. On the other hand, the hyperactivated $\mathrm{NF}-\kappa \mathrm{B}$ pathway contributed to EMT properties by enhancing MMP-2 syntheses in melanoma. These results demonstrate that the silencing of Bmi-1 suppressed melanoma invasion via the regulation of PTEN/Akt/NF- $\kappa \mathrm{B} / \mathrm{MMP}-2$ in A375 cells.

\section{Discussion}

Evidence has demonstrated that EMT plays a crucial role in the invasion and metastasis in cancers $(17,18)$. The EMT process occurs at the invasive front and produces single migratory cells with increased vimentin and loss of E-cadherin in colon and breast carcinoma, and melanoma (17-19). Moreover, there are other events that are highly relevant to EMT-promoting tumor progression, such as acceleration of proliferation and resistance to cell death, senescence, chemotherapy and immunotherapy, as well as conferring cancer cells with stem cell-like properties $(17,20-22)$.
Bmi-1 plays an essential role in the self-renewal of stem cells and its overexpression is involved in the proliferation, metastatic behavior and drug resistance of melanoma cells $(5,6,8,10,11,13,14)$. Overexpression of Bmi-1 induces EMT and promotes cell proliferation in oral epithelial cells (23). Previous studies have demonstrated that suppressing the EMT process could inhibit cell proliferation and tumor growth in breast cancer and squamous cell carcinoma $(22,24)$. Wound-healing assays have shown an obvious decrease in cell migration ability after the silencing of Bmi-1 in WM115A melanoma cells (14).

In the present study, the silencing of Bmi-1 significantly reduced the invasiveness of A375 cells in vitro as evidenced by observations of cell morphology and Matrigel invasion assays. Further studies focused on the change in the EMT process in the Bmi-1-silenced A375 melanoma cells. Compared with the A375-vector cells, EMT-related epithelial markers (E-cadherin and $\alpha$-catenin) were increased and EMT-related mesenchymal markers (vimentin and $\mathrm{N}$-cadherin) were decreased in the A375-Bmi-1-shRNA cells. Our results confirmed that Bmi-1 was crucial to inducing melanoma EMT and enhancing invasiveness in vitro.

Moreover, compared with controls, the average volume and weight of xenograft tumors formed by the A375-Bmi-1-shRNA cells were reduced in the late stages, which suggested that tumorigenesis ability decreased when Bmi-1 was silenced in the A375 cells. To analyze tumorigenesis differences between the two groups, we detected the expression of Ki67 (data not shown) and found that it was decreased in tumors formed by the A375-Bmi-1-shRNA cells compared to the control group. This confirmed that silencing of Bmi-1 actually inhibited the self-renewing process of melanoma cells, however, there was no marked difference in visible tumor mass between the two groups until the third week. Moreover, necrosis was much more obvious in tumors formed by the A375-vector cells compared to those formed by the A375-Bmi-1-shRNA cells. Necrosis may have resulted from the rapid growth of tumors 
without abundant blood vessel supply. We speculated that the reversal of EMT induced by silencing Bmi-1 in the A375 cells contributed more significantly to inhibiting tumor expansion.

In accordance with the detection in vitro, the expression of epithelial markers (E-cadherin and $\alpha$-catenin) was increased and mesenchymal markers (vimentin and $\mathrm{N}$-cadherin) significantly decreased in xenografts tissues formed by the A375-Bmi-1-shRNA cells. Based on these results, we proposed that Bmi-1 silencing weakened the invasive potential by suppressing EMT in A375 cells, which led to the delay of tumor graft growth and expansion. These data implied that the tumor mass expansion could be induced not only by cell proliferation, but also by EMT.

Different pathways (Fig. 5) are involved in the Bmi-1 regulation of EMT in various types of cancer $(4,8,25-27)$. However, the mechanism by which Bmi-1 induces EMT in melanoma is not clear. In the present study, we also addressed how Bmi-1 mediated EMT in melanoma. The tumor suppressor PTEN plays a significant role in cell cycle arrest, regulation of cell adhesion, migration and proliferation (4). PTEN regulates the switch from E- to N-cadherin and activates the PTEN/PI3K/Akt pathways in melanoma $(4,8,28)$. Furthermore, activation of Akt has been shown to activate the $\mathrm{NF}-\kappa \mathrm{B}$ pathway, which could directly regulate EMT in melanoma (4).

NF- $\kappa \mathrm{B}$ upregulates MMPs leading to EMT and malignant characteristics have been reported in breast cancers, osteosarcoma and glioma $(27,29,30)$. MMPs degrade structural components of the extracellular matrix, contribute to EMT, and promote tumor invasion and metastasis (7,31). MMP-2 plays an important role in melanoma invasion and correlates with progression and survival (7).

Whether the PTEN/PI3K/Akt/NF- $\mathrm{B} / \mathrm{MMP}$ pathway participates in Bmi-1-induced EMT in melanoma cells has not been previously elucidated. In the present study, Bmi-1 knockdown increased PTEN and reduced the levels of p-Akt, p-NF- $\kappa$ B and MMP-2, suggesting that the PTEN/PI3K/ $\mathrm{Akt} / \mathrm{NF}-\kappa \mathrm{B} / \mathrm{MMP}$ pathway participated in Bmi-1 regulation of EMT.

In summary, the present study demonstrates that Bmi-1 plays a critical role in the proliferation and metastasis of melanoma. Silencing of Bmi-1 significantly reversed EMT in melanoma in vitro and in vivo via the suppression of the the $\mathrm{PTEN} / \mathrm{Akt} / \mathrm{NF}-\kappa \mathrm{B}$ pathway, accompanied by MMP-2 reduction. Hence, the targeting of Bmi-1 plays a potential role in the treatment of melanoma.

\section{Acknowledgements}

The present study was supported by the National Natural Science Foundation of China (no. 81372912) and the Northwest Hospital Research Fund.

\section{References}

1. Bedogni B, Warneke JA, Nickoloff BJ, Giaccia AJ and Powell MB Notch1 is an effector of Akt and hypoxia in melanoma development. J Clin Invest 118: 3660-3670, 2008.

2. Sinnberg T, Lasithiotakis K, Niessner H, Schittek B, Flaherty KT, Kulms D, Maczey E, Campos M, Gogel J, Garbe C, et al: Inhibition of PI3K-AKT-mTOR signaling sensitizes melanoma cells to cisplatin and temozolomide. J Invest Dermatol 129: 1500-1515, 2009.
3. Hu Z, Fan H, Lv G, Zhou Q, Yang B, Zheng J and Cao W: 5-Aminolevulinic acid-mediated sonodynamic therapy induces anti-tumor effects in malignant melanoma via p53-miR-34a-Sirt1 axis. J Dermatol Sci 79: 155-162, 2015.

4. Lin K, Baritaki S, Militello L, Malaponte G, Bevelacqua Y and Bonavida B: The role of B-RAF mutations in melanoma and the induction of EMT via dysregulation of the NF- $\kappa \mathrm{B} /$ Snail/RKIP/PTEN circuit. Genes Cancer 1: 409-420, 2010.

5. Siddique HR and Saleem M: Role of BMI1, a stem cell factor, in cancer recurrence and chemoresistance: Preclinical and clinical evidences. Stem Cells 30: 372-378, 2012.

6. Dong P, Kaneuchi M, Watari H, Hamada J, Sudo S, Ju J and Sakuragi N: MicroRNA-194 inhibits epithelial to mesenchymal transition of endometrial cancer cells by targeting oncogene BMI-1. Mol Cancer 10: 99, 2011.

7. Liu F, Gomez Garcia AM and Meyskens FL Jr: NADPH oxidase 1 overexpression enhances invasion via matrix metalloproteinase- 2 and epithelial-mesenchymal transition in melanoma cells. J Invest Dermatol 132: 2033-2041, 2012.

8. Song LB, Li J, Liao WT, Feng Y, Yu CP, Hu LJ, Kong QL, $\mathrm{Xu}$ LH, Zhang X, Liu WL, et al: The polycomb group protein Bmi-1 represses the tumor suppressor PTEN and induces epithelial-mesenchymal transition in human nasopharyngeal epithelial cells. J Clin Invest 119: 3626-3636, 2009.

9. Ota I, Masui T, Kurihara M, Yook JI, Mikami S, Kimura T, Shimada K, Konishi N, Yane K, Yamanaka T, et al: Snail-induced EMT promotes cancer stem cell-like properties in head and neck cancer cells. Oncol Rep 35: 261-266, 2016.

10. Guo BH, Feng Y, Zhang R, Xu LH, Li MZ, Kung HF, Song LB and Zeng MS: Bmi-1 promotes invasion and metastasis, and its elevated expression is correlated with an advanced stage of breast cancer. Mol Cancer 10: 10, 2011.

11. Song LB, Zeng MS, Liao WT, Zhang L, Mo HY, Liu WL, Shao JY, Wu QL, Li MZ, Xia YF, et al: Bmi-1 is a novel molecular marker of nasopharyngeal carcinoma progression and immortalizes primary human nasopharyngeal epithelial cells. Cancer Res 66: 6225-6232, 2006.

12. Xu X,Liu Y, Su J, Li D, Hu J, Huang Q, Lu M, Liu X, Ren J, Chen W, et al: Downregulation of Bmi-1 is associated with suppressed tumorigenesis and induced apoptosis in $\mathrm{CD} 44^{+}$nasopharyngeal carcinoma cancer stem-like cells. Oncol Rep 35: 923-931, 2016.

13. Mihic-Probst D, Kuster A, Kilgus S, Bode-Lesniewska B, Ingold-Heppner B, Leung C, Storz M, Seifert B, Marino S, Schraml P, et al: Consistent expression of the stem cell renewal factor BMI-1 in primary and metastatic melanoma. Int $\mathbf{J}$ Cancer 121: 1764-1770, 2007.

14. Liu S, Tetzlaff MT, Cui R and Xu X: miR-200c inhibits melanoma progression and drug resistance through down-regulation of BMI-1. Am J Pathol 181: 1823-1835, 2012.

15. Madonna G, Ullman CD, Gentilcore G, Palmieri G and Ascierto PA: NF- $\kappa \mathrm{B}$ as potential target in the treatment of melanoma. J Transl Med 10: 53, 2012.

16. Xia D, Srinivas H, Ahn YH, Sethi G, Sheng X, Yung WK, Xia Q, Chiao PJ, Kim H, Brown PH, et al: Mitogen-activated protein kinase kinase- 4 promotes cell survival by decreasing PTEN expression through an NF kappa B-dependent pathway. J Biol Chem 282: 3507-3519, 2007.

17. Thiery JP, Acloque H, Huang RY and Nieto MA: Epithelialmesenchymal transitions in development and disease. Cell 139: 871-890, 2009

18. Gheorgheosu D, Jung M, Ören B, Schmid T, Dehelean C, Muntean D and Brüne B: Betulinic acid suppresses NGAL-induced epithelial-to-mesenchymal transition in melanoma. Biol Chem 394: 773-781, 2013.

19. Ronca R, Di Salle E, Giacomini A, Leali D, Alessi P, Coltrini D, Ravelli C, Matarazzo S, Ribatti D, Vermi W, et al: Long pentraxin-3 inhibits epithelial-mesenchymal transition in melanoma cells. Mol Cancer Ther 12: 2760-2771, 2013.

20. Micalizzi DS, Farabaugh SM and Ford HL: Epithelialmesenchymal transition in cancer: Parallels between normal development and tumor progression. J Mammary Gland Biol Neoplasia 15: 117-134, 2010.

21. Xie G, Ji A, Yuan Q, Jin Z, Yuan Y, Ren C, Guo Z, Yao Q, Yang K, Lin X, et al: Tumour-initiating capacity is independent of epithelial-mesenchymal transition status in breast cancer cell lines. Br J Cancer 110: 2514-2523, 2014.

22. Geng S, Guo Y, Wang Q, Li L and Wang J: Cancer stem-like cells enriched with CD29 and CD44 markers exhibit molecular characteristics with epithelial-mesenchymal transition in squamous cell carcinoma. Arch Dermatol Res 305: 35-47, 2013. 
23. Qiao B, Chen Z, Hu F, Tao Q and Lam AK: BMI-1 activation is crucial in hTERT-induced epithelial-mesenchymal transition of oral epithelial cells. Exp Mol Pathol 95: 57-61, 2013.

24. Fang X, Cai Y, Liu J, Wang Z, Wu Q, Zhang Z, Yang CJ, Yuan L and Ouyang G: Twist 2 contributes to breast cancer progression by promoting an epithelial-mesenchymal transition and cancer stem-like cell self-renewal. Oncogene 30: 4707-4720, 2011.

25. Yang MH, Hsu DS, Wang HW, Wang HJ, Lan HY, Yang WH, Huang CH, Kao SY, Tzeng CH, Tai SK, et al: Bmil is essential in Twist1-induced epithelial-mesenchymal transition. Nat Cell Biol 12: 982-992, 2010.

26. Ansieau S, Bastid J, Doreau A, Morel AP, Bouchet BP, Thomas C, Fauvet F, Puisieux I, Doglioni C, Piccinin S, et al: Induction of EMT by twist proteins as a collateral effect of tumor-promoting inactivation of premature senescence. Cancer Cell 14: 79-89, 2008.

27. Jiang L, Wu J, Yang Y, Liu L, Song L, Li J and Li M: Bmi-1 promotes the aggressiveness of glioma via activating the NF-kappaB/MMP-9 signaling pathway. BMC Cancer 12: 406, 2012.
28. Hao L, Ha JR, Kuzel P, Garcia E and Persad S: Cadherin switch from $\mathrm{E}$ - to $\mathrm{N}$-cadherin in melanoma progression is regulated by the PI3K/PTEN pathway through Twist and Snail. Br J Dermatol 166: 1184-1197, 2012.

29. Felx M, Guyot MC, Isler M, Turcotte RE, Doyon J, Khatib AM, Leclerc S, Moreau A and Moldovan F: Endothelin-1 (ET-1) promotes MMP-2 and MMP-9 induction involving the transcription factor NF-kappaB in human osteosarcoma. Clin Sci 110: 645-654, 2006

30. Cichon MA and Radisky DC: ROS-induced epithelial-mesenchymal transition in mammary epithelial cells is mediated by NF-kB-dependent activation of Snail. Oncotarget 5: 2827-2838, 2014.

31. Radisky DC, Levy DD, Littlepage LE, Liu H, Nelson CM, Fata JE, Leake D, Godden EL, Albertson DG, Nieto MA, et al: Raclb and reactive oxygen species mediate MMP-3-induced EMT and genomic instability. Nature 436: 123-127, 2005. 